\title{
Re-clustering and profiling of digestive system tumors according to microenvironment components
}

\section{Yongwei Wang}

Chengde Medical College: Chengde Medical University

\section{Sen Guo}

Chengde Medical College: Chengde Medical University

\section{Zhihong Chen}

Chengde Medical College: Chengde Medical University

\section{Bing Bai}

Chengde Medical College: Chengde Medical University

\section{Shuo Wang}

Chengde Medical College: Chengde Medical University

\section{Yaxian Gao ( $\nabla$ yaxiangao@163.com )}

Chengde Medical College: Chengde Medical University https://orcid.org/0000-0003-2650-9609

\section{Primary research}

Keywords: TCGA, digestive system tumors, immune, microenvironment

Posted Date: September 22nd, 2020

DOI: https://doi.org/10.21203/rs.3.rs-78206/v1

License: (1) This work is licensed under a Creative Commons Attribution 4.0 International License. Read Full License

Version of Record: A version of this preprint was published at Frontiers in Oncology on February 10th, 2021. See the published version at https://doi.org/10.3389/fonc.2020.607742. 


\section{Abstract}

Backgrounds

Immunotherapy has become the most promising therapy in digestive system tumors besides conventional chemotherapy and radiotherapy. However, only a few patients could benefit from this kind of immunotherapy such as immune checkpoint blocking (ICB) treatment. It is urgent to screening and profiling the subgroups of patients with different responsiveness to ICB treatment.

Methods

This study carried out analysis on digestive system tumor patients from the Cancer Genome Atlas (TCGA) cohorts. The analyses were mainly performed by GraphPad Prism 7 and R language.

Results

We have quantified the microenvironmental components of eight digestive system tumor patients in TCGA cohort and evaluated their clinical value. We re-clustered patients based on the characteristics of the microenvironment composition and divided these patients into 6 clusters. The difference between these 6 clusters were profiled including survival conditions, enriched biological process, genomic mutation and microenvironment traits. We also evaluated the response heterogeneity in ICB cohorts grouping by microenvironmental factors.

Conclusions

Cluster 3 was the most immune related cluster with high infiltration of non-tumor components and poor survival status, which was also exhibited an inhibitory immune status. High stromal score could represent cluster 3 and indicated a poor response in ICB cohorts.

\section{Background}

Digestive system tumors are the most common type of tumors with rapid malignant progression[1]. Even after the patients were treated with standard radiotherapy and chemotherapy, the prognosis was still not very satisfactory [2, 3]. One important reason is that the invisible nature of digestive system tumors makes it difficult to be detected early. While the tumors were often founded when the symptoms are obvious, unfortunately, it is often in the malignant advanced stage at this period. On the other hand, the metastasis and recurrent trait of digestive system tumors makes it difficult for conventional treatment programs to exert maximum effectiveness[4-8].

In recent years, more and more researches focus on the importance of tumor microenvironment in driving malignancy, including digestive system tumors[9-12]. Most previous studies only focused on tumor cells themselves and their internal mechanisms, but in fact, there exists mutual communication and regulation between tumor cells and other components of the microenvironment[13-15]. Tumor cells could 
reprogram their surrounding immune microenvironment and stromal microenvironment into a pro-tumor microenvironment through paracrine mechanisms. The reprogrammed microenvironment could facilitate the malignant phenotype of tumor cells such as proliferation, invasion, migration, pro-vasculogenic effect, etc[16]. Meanwhile, increasing evidences suggested that the disorganized microenvironment may contributing to the phenomenon that tumor cells escape from the conventional intervention treatment, such as chemotherapy, radiotherapy, anti-vasculogenic therapy as well as some classical molecular targets therapy[17-19].

Within these years, more and more studies have begun to focus on immunotherapy, which was considered as a promising therapy in the near future, extensive basic and preclinical research have been carried out. Among them, the most valuable and potential immune regulatory point blocking therapy (ICB) has achieved significant effects in inhibiting the malignant progression of tumors including digestive system tumors, while clinical trial results show that there is only a part of tumor patients response well to ICB therapy[20]. The difference and complexity of microenvironmental components may partially explain the heterogeneity of ICB immunotherapy response among one kind of tumor patients[18]. Considering the fact that there are currently few studies on the comprehensive profiling and clustering of the microenvironmental components of digestive system tumors, we have quantitatively scored and then displayed the microenvironmental components of eight digestive system tumor patients in TCGA cohort. Meanwhile, we evaluated the clinical value of corresponding cell components in specific cancer, we found that some cell components were often accompanied with poor prognosis such as neutrophils, fibroblasts, and endothelial cells. Subsequently, we re-clustered patients with digestive system tumors based on microenvironmental components, and conducted in-depth analysis including clinical prognostic difference, matrix and genomic's level, enriched biological processes, and microenvironmental component characteristics. In addition, we found that the stromal score robustly enhanced in cluster 3 subgroup, which was consistently correlated with multiple negative immune cell components. We proposed that the inhibitory immune status may characterized by high stromal scores. In the Imivor210 database, the response rate of ICB immunotherapy for patients with high stromal scores was significantly limited, which confirmed the relationship between stromal components and the inhibitory immune microenvironment.

\section{Methods}

\section{Data acquisition}

The RNA sequencing and clinical information of 1526 patients were downloaded from the UCSC website(https://genome.ucsc.edu/). These six kinds of tumors' mutation data (MAF file) was downloaded from https://portal.gdc.cancer.gov/ and somatic copy number variation (SCNA) information was acquired from Firehose (http://gdac.broadinstitute.org/).

\section{The quantification of microenvironment components and $\mathrm{K}$ - means clustering analysis}


ESTIMATE R package was performed to calculate stromal and immune scores and tumor purity was calculated according to the formula from Yoshihara and colleagues[21]. The relative immune cells proportions were calculated based on the CIBERSORT algorithm[22]. MCP counter was conducted to calculate the enrichment of several critical immune and stromal cell components[23]. The cluster analyses based on the MCP counter results were performed by consensus unsupervised analysis according to the ClusterProfiler R package[24].

\section{Differential enriched biological process and driver mutations}

Limma R package was used to calculate the differential genes among different clusters. Gene set variation analysis (GSVA) was conducted to explore differential signaling pathway among different clusters[25]. Maftools R package was performed to illustrate significant differential mutation information of driver genes.

\section{Statistical analysis}

R 3.6.1 (https://www.r-project.org/)and GraphPad Prism 7 were mainly used for statistical analysis. Kaplan-Meier survival analysis was used to evaluate the prognostic value. The receiver operating characteristic (ROC) curve was made using GraphPad Prism 7 software. Student's t test was performed to analysis differential expression. Survival curves were exported by GraphPad Prism 7. Two-tailed $p$ value < 0.05 was termed as significant.

\section{Results}

\section{Overall profiling of the digestive system tumor microenvironment components and clinical value}

We first calculated the microenvironmental components of each digestive system tumor patient through the MCP counter package and performed a visual exhibition (Fig. 1A), we found that the content of fibroblasts is the most enriched and the content of NK cells is less enriched. Similarly, by drawing a heat map, we can clearly find that in the digestive system tumor microenvironment, the two main components of fibroblasts and vascular endothelial cells are more enriched in the microenvironment composition, and the main immune components are monocytes (Fig. 1B). Next, we calculated the impact of each microenvironment component on the survival prognosis of tumor patients through univariate cox regression analysis in each type of tumor. The survival-related microenvironment component of stomach adenocarcinoma (STAD) occupied the majority (Fig. 1C). We used the Log-rank test method to draw survival curves of the microenvironment components, aiming to identified survival-related components in different tumors. We found that the survival-related microenvironment components in liver hepatocellular carcinoma (LIHC), pancreatic adenocarcinoma (PAAD) and rectum adenocarcinoma (READ) are not effective under the log-rank test. While in STAD, COX suggests that neutrophils, fibroblasts and 
endothelial cells associated with poor prognosis, which were also significantly related to the prognosis under the log-rank test.

\section{Re-clustering digestive system tumor patients based on microenvironmental components}

We performed $\mathrm{K}$ means unsupervised clustering of patients with digestive system tumors based on the characteristics of the microenvironmental components calculated by MCP (Fig. 2A-B), and the results showed that the 6 types of discrimination were the best. We analyzed the proportions of the six categories in each type of digestive system tumors. The results showed that the COAD and READ had a relative average distribution among these six clusters, while cluster 4 in ESCA was relatively enriched, and cluster 6 in LIHC was the main component. Cluster 1 was dominant in PAAD, and cluster 3 had the highest proportion in STAD (Fig. 2C). We further performed a Sankey diagram to depict the correspondence between cancer species and clusters (Fig. 2D). Moreover, we described the main nontumor cell components in different clusters and found that cluster 3 contains the highest content of immune cells and stromal cells, while cluster 6 contains relatively low content (Fig. 2E). Survival analysis suggests that cluster 1 and cluster 3 , which have a high proportion of microenvironmental components, have a relatively poor prognosis, while cluster 6 subgroup with a low proportion of non-tumor cells has a relatively good prognosis (Fig. $2 \mathrm{~F}-\mathrm{H}$ ).

\section{Profiling of cluster-related mutations at the genome level}

In order to compare the differences between different clusters at the genome level, we obtained the SNP mutation data of these 6 digestive system tumors. Since the cluster 3 was accompanied by a higher content of non-tumor microenvironment components, and cluster 3 in STAD is relatively high, in order to exclude the influence of the tumor type, we have analyzed the classic tumor driver gene mutations in the cluster 3 and non-cluster 3 subgroups of STAD. The results showed that the cluster 3 subgroup of STAD patients was accompanied by a lower TP53 mutation rate and a higher LRP1B mutation rate, suggesting a potential upstream mechanism for the poor prognosis and increased infiltration of non-tumor components of cluster 3 . Similarly, due to the relatively large proportion of cluster 6 in LIHC, we analyzed the classic driver gene genome mutations of cluster 6 and non-cluster 6 subgroups of LIHC patients, we found that the cluster 6 subgroups of LIHC were accompanied by higher CTNNB1 mutations and TTN mutation. As PAAD occupied with a large proportion of cluster 1 In PAAD, we compared the patients, we compared the genomic differences between the cluster 1 subgroup and the non-cluster 1 subgroup, we found that the $\mathrm{C} 1$ subgroup of PAAD was accompanied by a higher mutation rate of KRAS and SMAD4, suggesting a potential mechanism for the poor prognosis of cluster 1 patients.

\section{Differential function enrichment analysis among clusters}

In order to explore the underlying mechanism of differences in clinical characteristics and survival conditions of patients in different clusters, we selected more than 70 classical tumor related pathways or 
critical biological processes, and calculated the corresponding SSGSEA score for each tumor patient, then we exhibited the results by using heatmap, we found that some pathways that regulate the malignant behavior of tumor cells and immune-related pathways are significantly enriched in cluster 3 , which characterized by high infiltration of non-tumor cells (Fig. 4A). Subsequently, we conducted a series of comparison from the aspect of cancer hallmarks. In terms of several classical metabolic pathways, the enrichment of cluster 6 was significantly higher than that of other clusters. This may be result from that cluster 6 was mainly composed by tumor cells (Fig. 4B-F). The level of DNA replication and mismatch repair of cluster 2 was significantly higher than that of the other clusters (Fig. 4G-H). Cluster 3 was focus on the interaction of cytokines and receptors, chemokines, TGF $\beta$ pathway, VEGF pathway and focal adhesion pathway, which further suggested that microenvironmental factors may lead to the unique clinical characteristics of cluster 3 patients.

\section{Cluster 3 is closely related to the characteristics of the immunosuppressive microenvironment}

In order to further evaluate the microenvironment characteristics of patients in cluster 3 , we performed Xcell analysis and displayed the results of each cluster subgroup with a heatmap. The results showed that the immune cell and stromal cell components in cluster 3 were robustly enriched, which included activated and suppressed immune cell components (Fig. 5A). Moreover, the microenvironmental score, immune score and stromal score in cluster 3 were significantly higher than other clusters (Fig. 5B-D). As for the classical inhibitory immune checkpoints, we found that PD1, PDL1 and CTLA4 molecules in cluster 3 and cluster 5 were significantly increased (Fig. 5E-G).

\section{Stromal score could be used to predict the response of anti- PD1/PDL1 treatment}

Based on the robustly enriched microenvironmental components and suppressive immune status of cluster 3, we proposed that tumor patients with cluster 3 traits may be insensitive to immunotherapy. We represented the characteristics of cluster 3 with microenvironment score, immune score and stromal score respectively, and then evaluated the effective response rate of different groups in the ICB immunotherapy cohorts. Firstly, in the Imvigor210 anti-PDL1 immunotherapy cohort, we found that when the microenvironment score and immune score were used as the stratified criteria, there is no significant difference in the immunotherapy response rate between the high and low score groups (Fig. 6A-B). Interestingly, when the stromal score was used as the distinction criteria, we found that the immunotherapy response rate in the high score group was significantly lower than that in the low-scoring group (Fig. 6C). Moreover, ROC curve showed that stromal score had a well predictive effect on the positive response rate (Fig. 6D). In addition, the stromal score in the response group was also significantly lower than the no response group (Fig. 6E). Similarly, in the GSE78220 anti-PD1 immunotherapy cohort, we got a similar conclusion, that is compared with the microenvironment score and immune score, the stromal score is more effective in distinguishing patients with positive treatment 
response (Fig. 6F-H). In addition, the stromal score has a good predictive effect on the positive response to ICB treatment. The stromal score of the positive response group was significantly lower than that of the unreacted group (Fig. 6I-J).

\section{Discussion}

Digestive system tumor patients account for more than $50 \%$ of tumor patients, which bring a great economic burden to individuals and society. Digestive system tumors were difficult to be detected early due to their strong compensatory ability and invisibility. Moreover, the therapeutic effects of digestive system tumors that in the middle and late stages were still not satisfactory after surgical resection and postoperative conventional radiotherapy or chemotherapy[1, 2]. Therefore, it is urgent to find a new treatment strategy. Immunotherapy has become the most promising option. However, some clinical trials of immune checkpoints blocking (ICB) have shown that only some cancer patients can benefit from it [20]. Therefore, screening and profiling the subgroups of patients with different responsiveness to ICB treatment will have significant clinical value.

Increasing studies have shown that the microenvironmental components of digestive system tumors are important factors affecting the poor prognosis and low response to treatment. The non-tumor cell components and tumor cell components in the microenvironment can mutually regulate and transform each other to accelerate the malignant progress of tumors[16]. Analyzing the microenvironment composition mode of different tumor patients can provide a certain reference value for the selection of the next treatment strategy. This study first quantified eight key microenvironment components of patients with digestive system tumors, and found that the two main stromal components of fibroblasts and vascular endothelial cells were prefer enriched, and the most enriched immune components were monocytes. We further evaluated the clinical value of these key microenvironmental components and found that fibroblasts, vascular endothelial cells and neutrophils are closely related to poor prognosis.

In the past, the classification of digestive system tumors was mainly based on clinical parameters such as tissue source, TNM stage and grade, etc. This study firstly clustered patients based on the characteristics of the microenvironment composition. We believe that similar microenvironment composition characteristics may have similar clinical characteristics. Based on the characteristics of the microenvironmental components calculated by MCP-Counter, we performed K means unsupervised clustering on patients with digestive system tumors, and divided these patients into 6 clusters. We quantified the main non-tumor cell components in different clusters and found that cluster 3 has the highest content of immune cells and stromal cells, and cluster 6 has a relatively low content. Survival analysis suggests that the prognosis of cluster 1 and cluster 3 were relatively poor, which have a higher proportion of microenvironmental components, while the prognosis of the cluster 6 those with a lower proportion of non-tumor cells is relatively well.

In addition, in order to explore the underlying mechanism of the differences of patients in different clusters, we quantified classical tumor driven biological process, and we found that in cluster 3 with high 
non-tumor cell components, some pathways that regulate the malignant behavior of tumor cells and immune-related pathways were significantly enriched. In addition, the cluster 3 was significantly enriched in microenvironment-related functions such as cytokine-receptor interactions, chemokines, TGFB pathway, VEGF pathway, and focal adhesion pathway, further suggested that microenvironmental factors may contribute to the unique clinical features of cluster 3 . Based on the significantly enriched microenvironmental components and suppressive immune status of cluster 3 , we speculate that tumor patients with cluster 3 characteristics may be insensitive to immunotherapy. We replaced the characteristics of cluster 3 with microenvironment score, immune score and stromal score respectively, and then tested the treatment response rate of different groups in the ICB immunotherapy cohorts. First of all, in the Imvigor210 anti-PDL1 immunotherapy cohort, we found that when the stromal score was used as the distinguishing standard, the immunotherapy response rate in the high score group was significantly lower than that in the low score group. The ROC curve showed that the stromal score had a good predictive effect on the positive response rate. Expectedly, the stromal score in the positive response group was also significantly lower than that in the un-response group. Similarly, in the GSE78220 antiPD1 immunotherapy cohort, we have also reached a similar conclusion that stromal score was more effective in distinguishing patients with positive response to ICB treatment.

In summary, the composition of the microenvironmental components of various tumors in the digestive system is heterogeneous. There is a subgroup of patients characterized with high stromal and immune components, which accompanied with a poor prognosis and insensitivity to ICB therapy. Our research provided new ideas for the precise diagnosis and treatment of digestive system tumor patients.

\section{Abbreviations}

ICB: Immune checkpoint blocking; TCGA:The Cancer Genome Atlas; SCNA:Somatic copy number variation; GSVA:Gene set variation analysis; ROC:Receiver operating characteristic; STAD:Stomach adenocarcinoma; LIHC:Liver hepatocellular carcinoma; PAAD:Pancreatic adenocarcinoma; READ:Rectum adenocarcinoma; COAD:Colon adenocarcinoma; ESCA:Esophageal adenocarcinoma.

\section{Declarations}

\section{Acknowledgements}

Not applicable

\section{Funding}

This work was supported by the Science and Technology Research Project for Colleges and Universities in Hebei Province (grant number: QN2016060, QN2015044); Launch Fund for high-level Talents Scientific Research of Chengde Medical College(grant number: 202002); Medical Scientific Research Project for Hebei Provincial Health and Family Planning Commission (grant number: 20160313, 20160310). 


\section{Author contributions}

Conception and design: Wang YW, Gao YX; data download and curation: Bai B, Wang S; methodology: Chen ZH; data analyses and interpretation: Wang YW, Guo S; manuscript writing and revision: Wang YW, Gao YX. All authors read and approved the final manuscript.

\section{Disclosure of conflict of interest}

None

\section{Ethics approval and consent to participate}

Not applicable

\section{Consent of publication}

Not applicable

\section{Data availability statement}

The RNA sequencing and clinical information of 1526 patients were downloaded from the UCSC website(https://genome.ucsc.edu/). These six kinds of tumors' mutation data (MAF file) was downloaded from https://portal.gdc.cancer.gov/ and somatic copy number variation (SCNA) information was acquired from Firehose (http://gdac.broadinstitute.org/).

\section{References}

1. Bray F, Ferlay J, Soerjomataram I, Siegel R, Torre L, Jemal A. Global cancer statistics 2018: GLOBOCAN estimates of incidence and mortality worldwide for 36 cancers in 185 countries. Cancer J Clin. 2018;68(6):394-424.

2. Kawaguchi Y, Vauthey J. The Landmark Series: Randomized Control Trials Examining Perioperative Chemotherapy and Postoperative Adjuvant Chemotherapy for Resectable Colorectal Liver Metastasis. Annals of surgical oncology 2020.

3. St James S, Bednarz B, Benedict S, Buchsbaum J, Dewaraja Y, Frey E, Hobbs R, Grudzinski J, Roncali E, Sgouros G, et al: Current Status of Radiopharmaceutical Therapy. International journal of radiation oncology, biology, physics 2020.

4. Dai W, Wang Y, Yang T, Wang J, Wu W, Gu J. Downregulation of exosomal CLEC3B in hepatocellular carcinoma promotes metastasis and angiogenesis via AMPK and VEGF signals. Cell communication signaling: CCS. 2019;17(1):113.

5. Ye K, Ouyang X, Wang Z, Yao L, Zhang G. SEMA3F Promotes Liver Hepatocellular Carcinoma Metastasis by Activating Focal Adhesion Pathway. DNA cell biology. 2020;39(3):474-83. 
6. Gu L, Liu Y, Jiang C, Sun L, Zhou H. Identification and clinical validation of metastasis-associated biomarkers based on large-scale samples in colon-adenocarcinoma. Pharmacological research. 2020;160:105087.

7. Liu J, Zhu Y, Ge C. LncRNA ZFAS1 promotes pancreatic adenocarcinoma metastasis via the RHOA/ROCK2 pathway by sponging miR-3924. Cancer cell international. 2020;20:249.

8. Zang D, Zhang C, Li C, Fan Y, Li Z, Hou K, Che X, Liu Y, Qu X. LPPR4 promotes peritoneal metastasis via Sp1/integrin a/FAK signaling in gastric cancer. American journal of cancer research. 2020;10(3):1026-44.

9. Cao D, Chen M, Zhang Q, Zhou Y, Zhang M, Mai S, Zhang Y, Chen M, Li X, Wang H. Identification of immunological subtypes of hepatocellular carcinoma with expression profiling of immunemodulating genes. Aging. 2020;12(12):12187-205.

10. Liu H, Ni S, Wang H, Zhang Q, Weng W. Charactering tumor microenvironment reveals stromal-related transcription factors promote tumor carcinogenesis in gastric cancer. Cancer medicine. 2020;9(14):5247-57.

11. Yang S, Liu T, Cheng Y, Bai Y, Liang G. Immune cell infiltration as a biomarker for the diagnosis and prognosis of digestive system cancer. Cancer Sci. 2019;110(12):3639-49.

12. Zhu X, Xie X, Zhao Q, Zhang L, Li C, Zhao D. Potential Prognostic Value and Mechanism of StromalImmune Signature in Tumor Microenvironment for Stomach Adenocarcinoma. BioMed research international. 2020;2020:4673153.

13. Navarro R, Tapia-Galisteo A, Martín-García L, Tarín C, Corbacho C, Gómez-López G, Sánchez-Tirado E, Campuzano S, González-Cortés A, Yáñez-Sedeño P, et al: TGF- $\beta$-induced IGFBP-3 is a key paracrine factor from activated pericytes that promotes colorectal cancer cell migration and invasion. Molecular oncology 2020.

14. Sperb N, Tsesmelis M, Wirth T. Crosstalk between Tumor and Stromal Cells in Pancreatic Ductal Adenocarcinoma. International journal of molecular sciences 2020, 21(15).

15. Mroweh M, Decaens T, Marche P, Macek Jilkova Z, Clément F. Modulating the Crosstalk between the Tumor and Its Microenvironment Using RNA Interference: A Treatment Strategy for Hepatocellular Carcinoma. International journal of molecular sciences 2020, 21(15).

16. Zhao W, Ajani J, Sushovan G, Ochi N, Hwang R, Hafley M, Johnson R, Bresalier R, Logsdon C, Zhang Z, et al. Galectin-3 Mediates Tumor Cell-Stroma Interactions by Activating Pancreatic Stellate Cells to Produce Cytokines via Integrin Signaling. Gastroenterology. 2018;154(5):1524-37.e1526.

17. Aronovich A, Moyal L, Gorovitz B, Amitay-Laish I, Naveh H, Forer Y, Maron L, Knaneh J, Ad-El D, Yaacobi D, et al: Cancer-Associated Fibroblasts in Mycosis Fungoides Promote Tumor Cell Migration and Drug Resistance via CXCL12/CXCR4. The Journal of investigative dermatology 2020.

18. Wang S, Li Y, Xing C, Ding C, Zhang H, Chen L, You L, Dai M, Zhao Y. Tumor microenvironment in chemoresistance, metastasis and immunotherapy of pancreatic cancer. American journal of cancer research. 2020;10(7):1937-53. 
19. Orme J, Huang H. Microenvironment-Mediated Resistance to Anti-Androgen Therapy. Cancer cell. 2020;38(2):155-7.

20. Lei Q, Wang D, Sun K, Wang L, Zhang Y. Resistance Mechanisms of Anti-PD1/PDL1 Therapy in Solid Tumors. Frontiers in cell developmental biology. 2020;8:672.

21. Bindea G, Mlecnik B, Tosolini M, Kirilovsky A, Waldner M, Obenauf A, Angell H, Fredriksen $T$, Lafontaine L, Berger A, et al. Spatiotemporal dynamics of intratumoral immune cells reveal the immune landscape in human cancer. Immunity. 2013;39(4):782-95.

22. Newman A, Liu C, Green M, Gentles A, Feng W, Xu Y, Hoang C, Diehn M, Alizadeh A. Robust enumeration of cell subsets from tissue expression profiles. Nature methods. 2015;12(5):453-7.

23. Becht E, Giraldo N, Lacroix L, Buttard B, Elarouci N, Petitprez F, Selves J, Laurent-Puig P, SautèsFridman $\mathrm{C}$, Fridman W, et al. Estimating the population abundance of tissue-infiltrating immune and stromal cell populations using gene expression. Genome biology. 2016;17(1):218.

24. Yu G, Wang L, Han Y, He Q. clusterProfiler: an R package for comparing biological themes among gene clusters. Omics: a journal of integrative biology. 2012;16(5):284-7.

25. Hänzelmann S, Castelo R, Guinney J. GSVA: gene set variation analysis for microarray and RNA-seq data. BMC Bioinform. 2013;14:7.

\section{Figures}


A

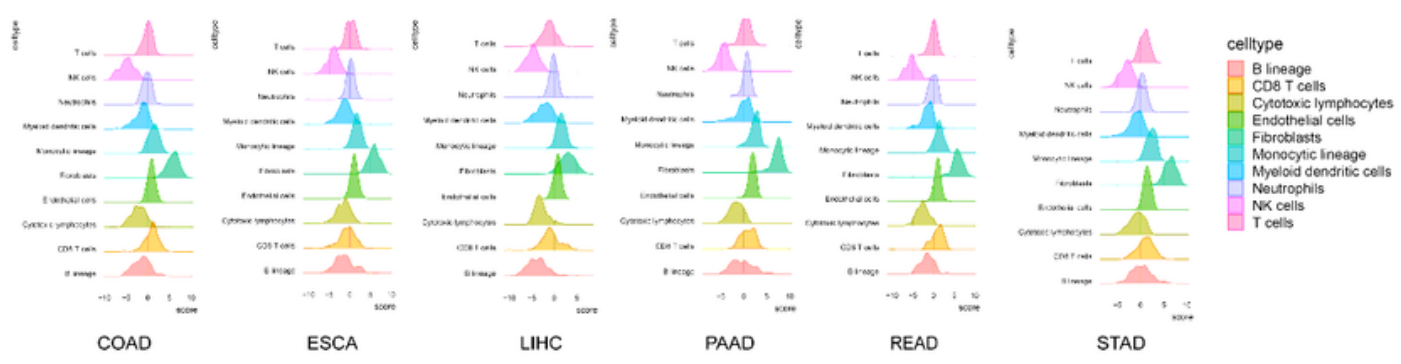

B

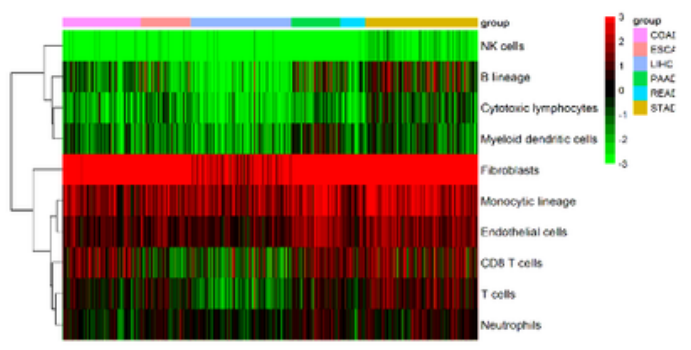

C TCGA RNA seq, STAD cohort

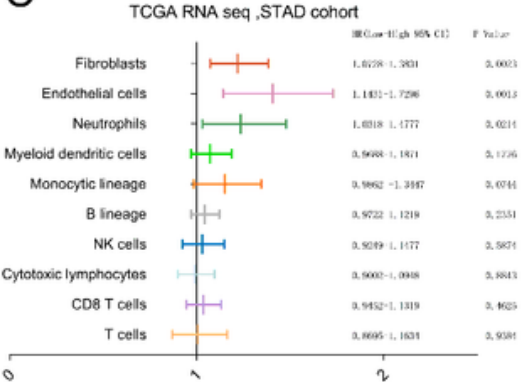

D

TCGA seq. LHHC cohort.CDB T cells

E

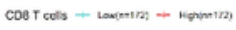

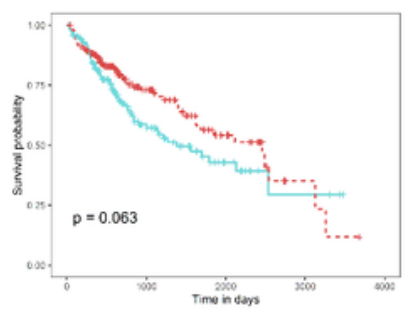

Number at risk

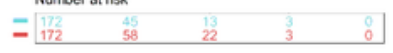

G

TCGA seq, STAD cohor, Neutrophits

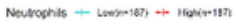

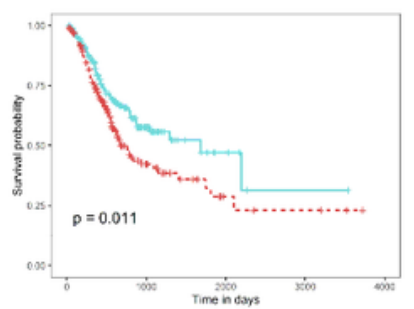

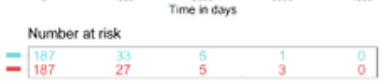

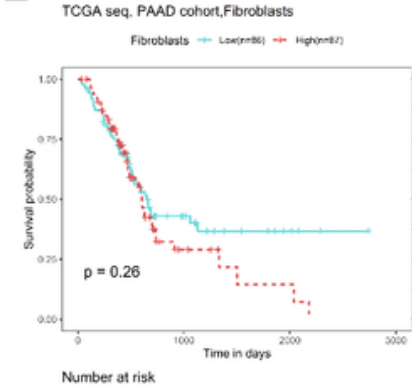

$\mathrm{F}$

TCGA seq, READ cohort,CDE T cells

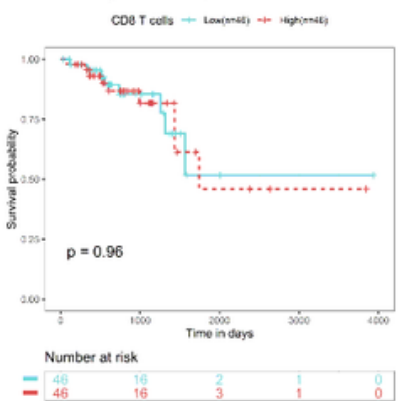

$=\begin{aligned} & \text { Number } \\ & 87\end{aligned}$

$\mathrm{H}$

TCGA seq, STAD cohort,Endothelial cells

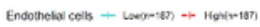
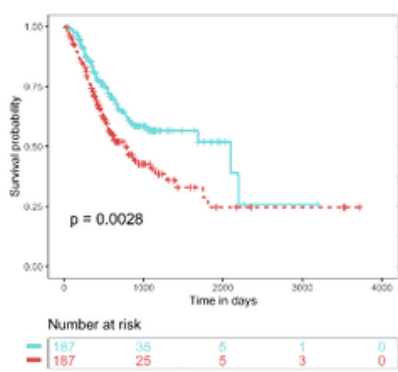

I

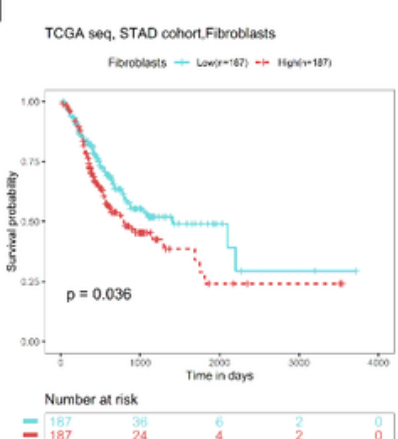

\section{Figure 1}

Overall description of the digestive system tumor microenvironment components and clinical value. (A) The landscape of microenvironmental components in digestive system cancers. (B) Heatmap of the MCP-counter results in these six cancers. (C) Univariate cox results of each cell component in STAD cohort. (D-I) The log-rank survival curve of some type of cells in specific cancer with prognostic value. 
A

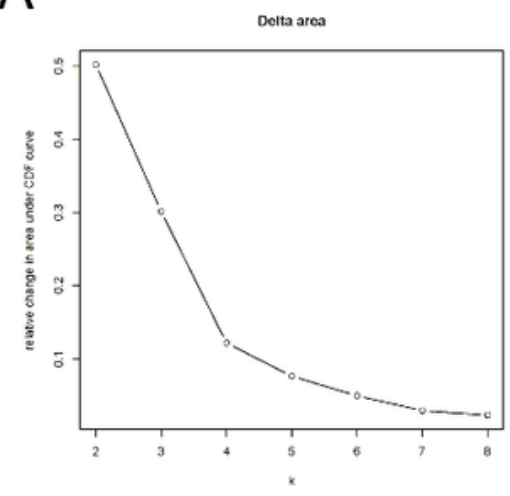

D

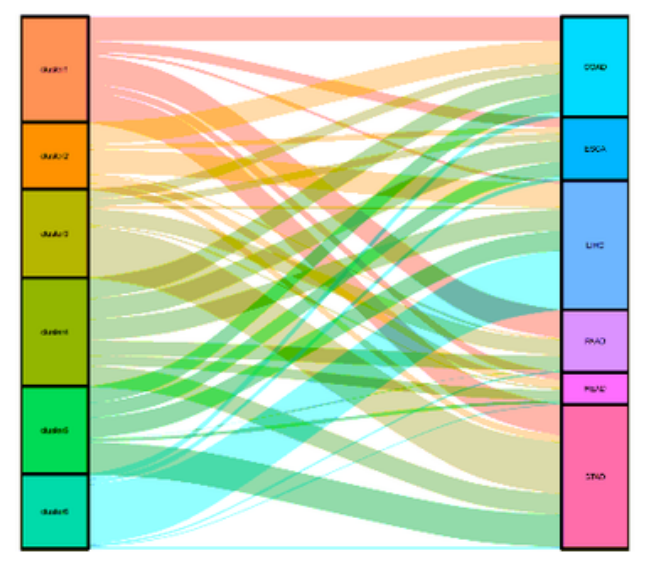

B

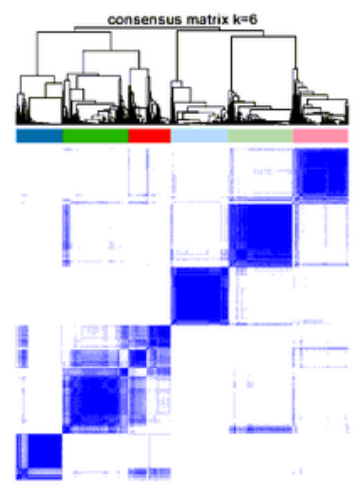

C

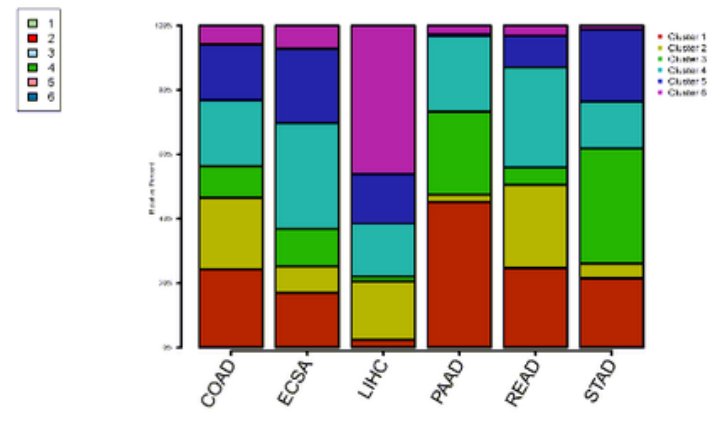

E

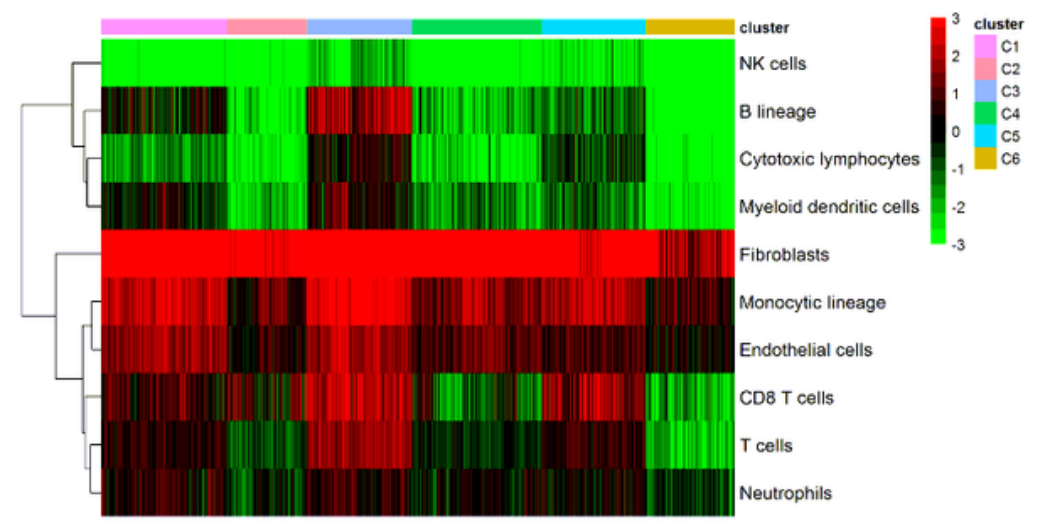

$\mathrm{F}$

G

TCGA Seq DSS

Cluster $+\operatorname{cis}(n=22 m+\cdot \operatorname{cs}(n=227)+\cos (n=228)$

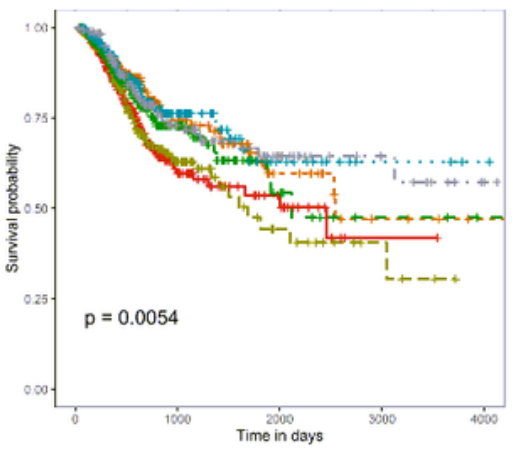

H TCGA Seq PFI

Cluster $+C(n=286)+\cdot \operatorname{csin}=2351+C \operatorname{cs}(n=2301$

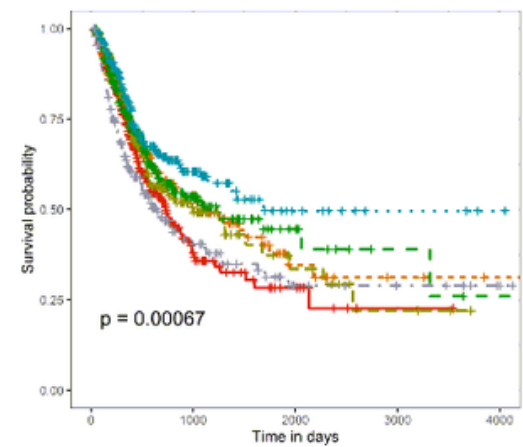

\section{Figure 2}

Re-clustering digestive system tumor patients based on microenvironmental components. (A-B) K means unsupervised clustering of patients with digestive system tumors based on the characteristics of the microenvironmental components. (C) The proportions of the six categories in each type of digestive system tumors. (D) Sankey diagram was performed to depict the correspondence between cancer species 
and clusters. (E) The heatmap of the microenvironmental components in these six clusters. $(\mathrm{F}-\mathrm{H})$ Survival analyses between these six clusters in OS, DSS and PFI.
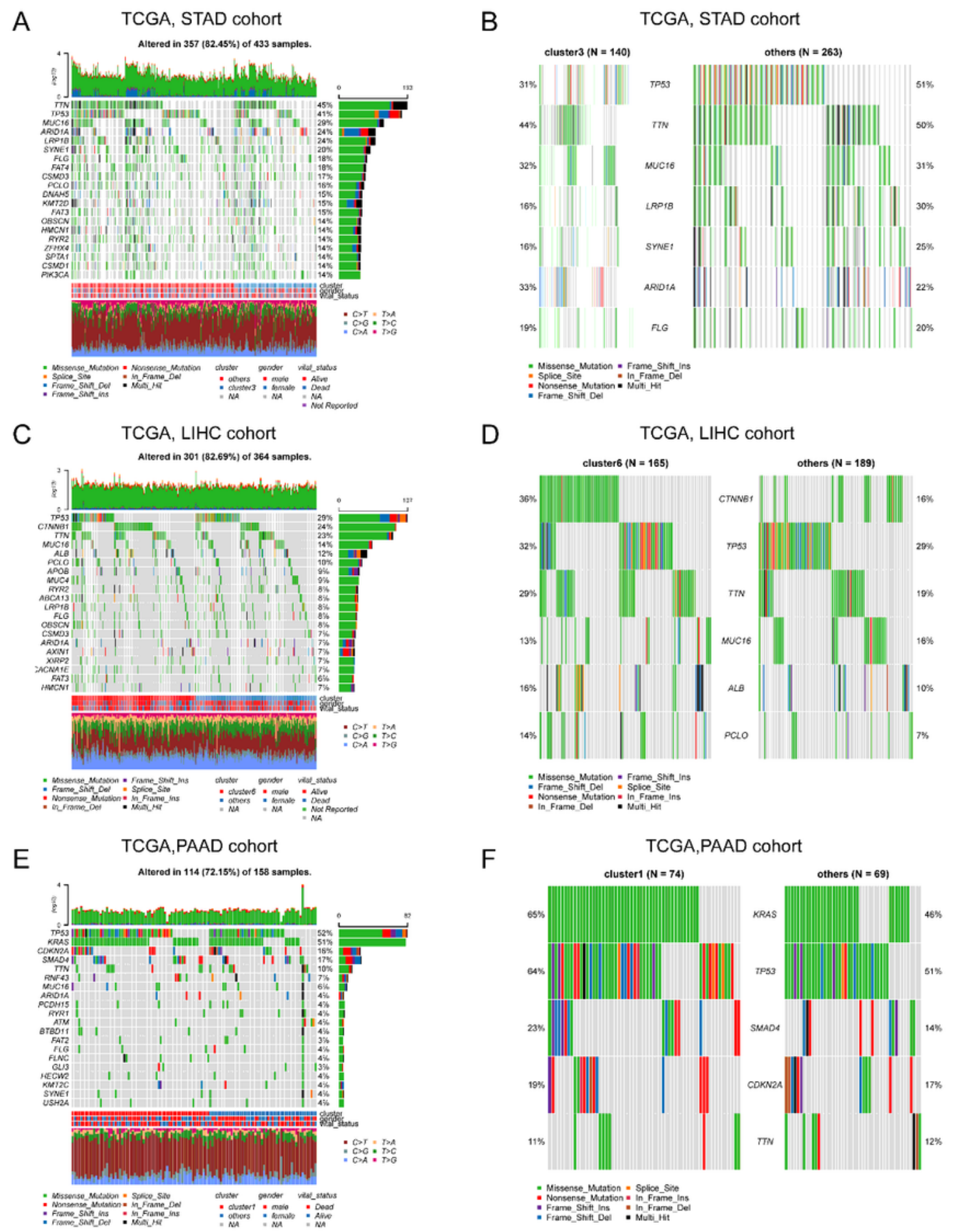

\section{Figure 3}

Profiling of cluster-related mutations at the genome level. (A) The landscape of classical driver gene mutations in the $\mathrm{C} 3$ and non-C3 clusters of STAD cohort. (B) The most different driver mutations between C3 and non-C3 clusters of STAD cohort. (C) The landscape of classical driver gene mutations in the C6 
and non-C6 clusters of $\mathrm{LIHC}$ cohort. (D) The most different driver mutations between $\mathrm{C} 6$ and non-C6 clusters of $\mathrm{LIHC}$ cohort. (E) The landscape of classical driver gene mutations in the $\mathrm{C} 1$ and non-C1 clusters of PAAD cohort. (F) The most different driver mutations between $\mathrm{C} 1$ and non-C1 clusters of PAAD cohort.
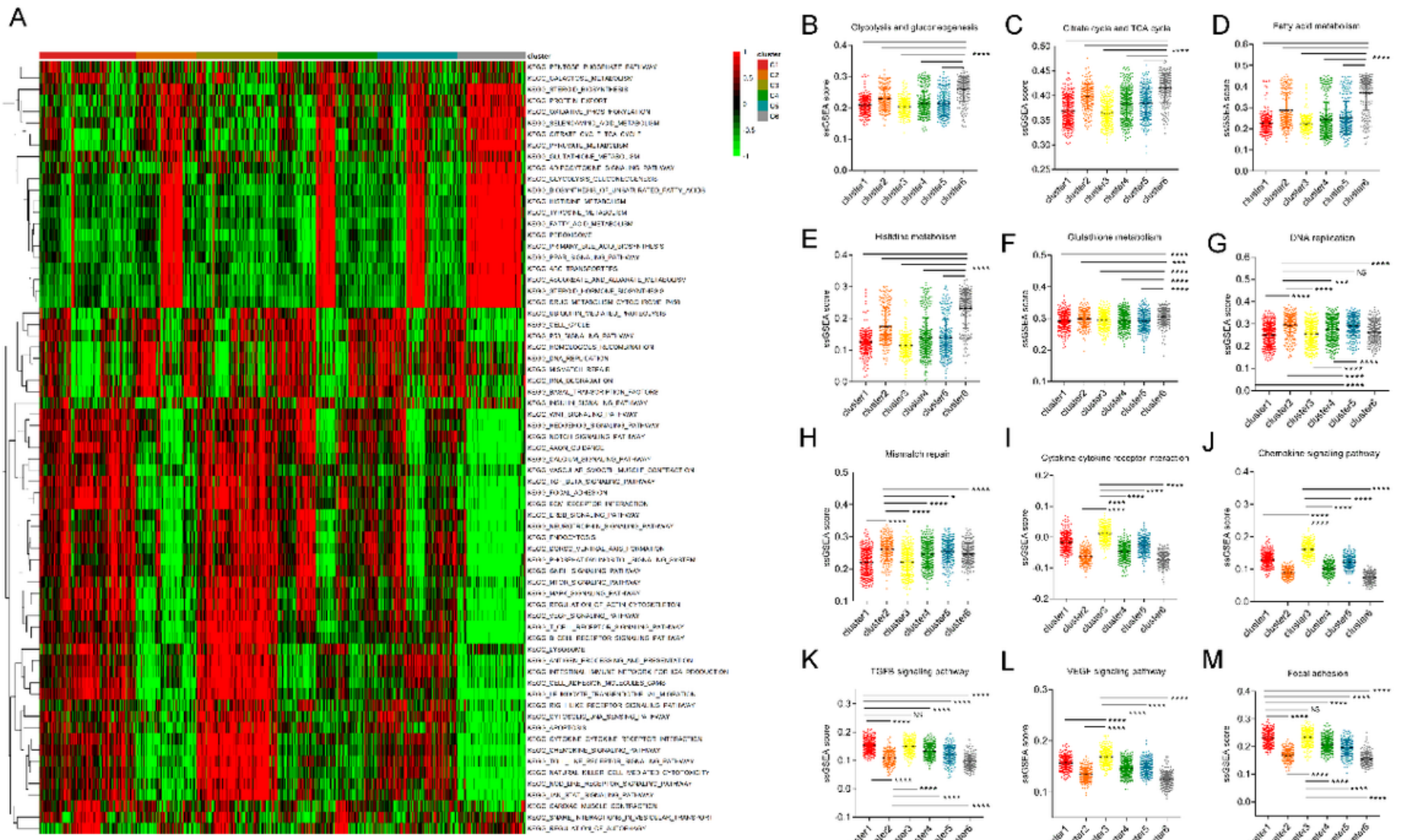

E
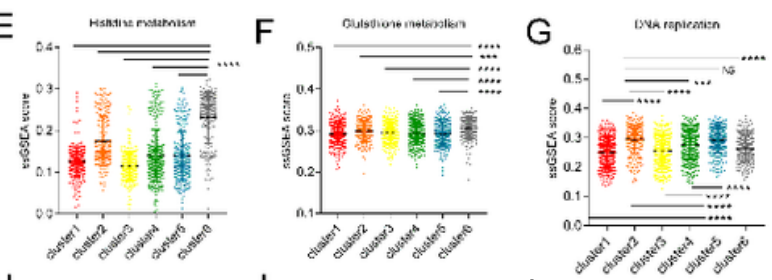

$\mathrm{H}$
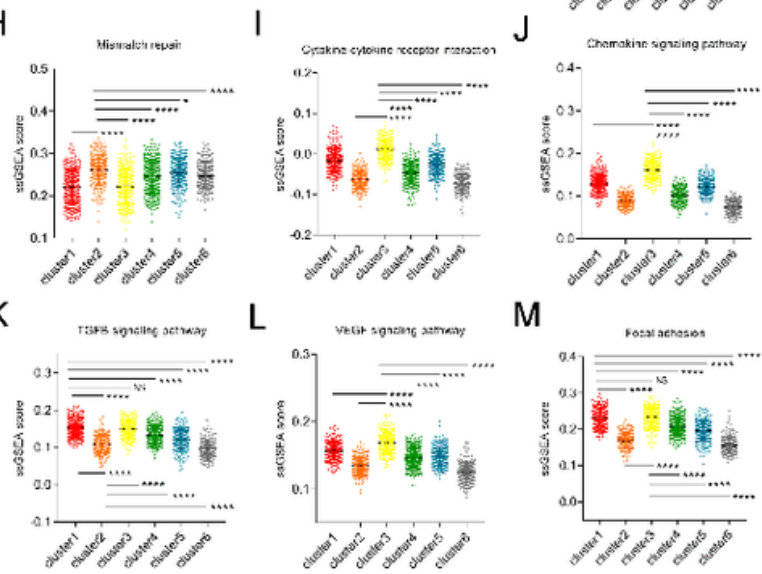

\section{Figure 4}

Differential function enrichment analysis among clusters. (A) More than 70 classical tumor-related pathways were quantified by ssGSEA method and exhibited in heatmap. (B-F) Several metabolic pathways enhanced in cluster 6 . (G-H) The level of DNA replication and mismatch repair of the cluster 2 is significantly higher than that of the other subgroups. (I-M) The cluster 3 was enriched with immune microenvironmental related terms such as the interaction of cytokines and receptors, chemokines, TGFB pathway, VEGF pathway and focal adhesion pathway, etc. 
A

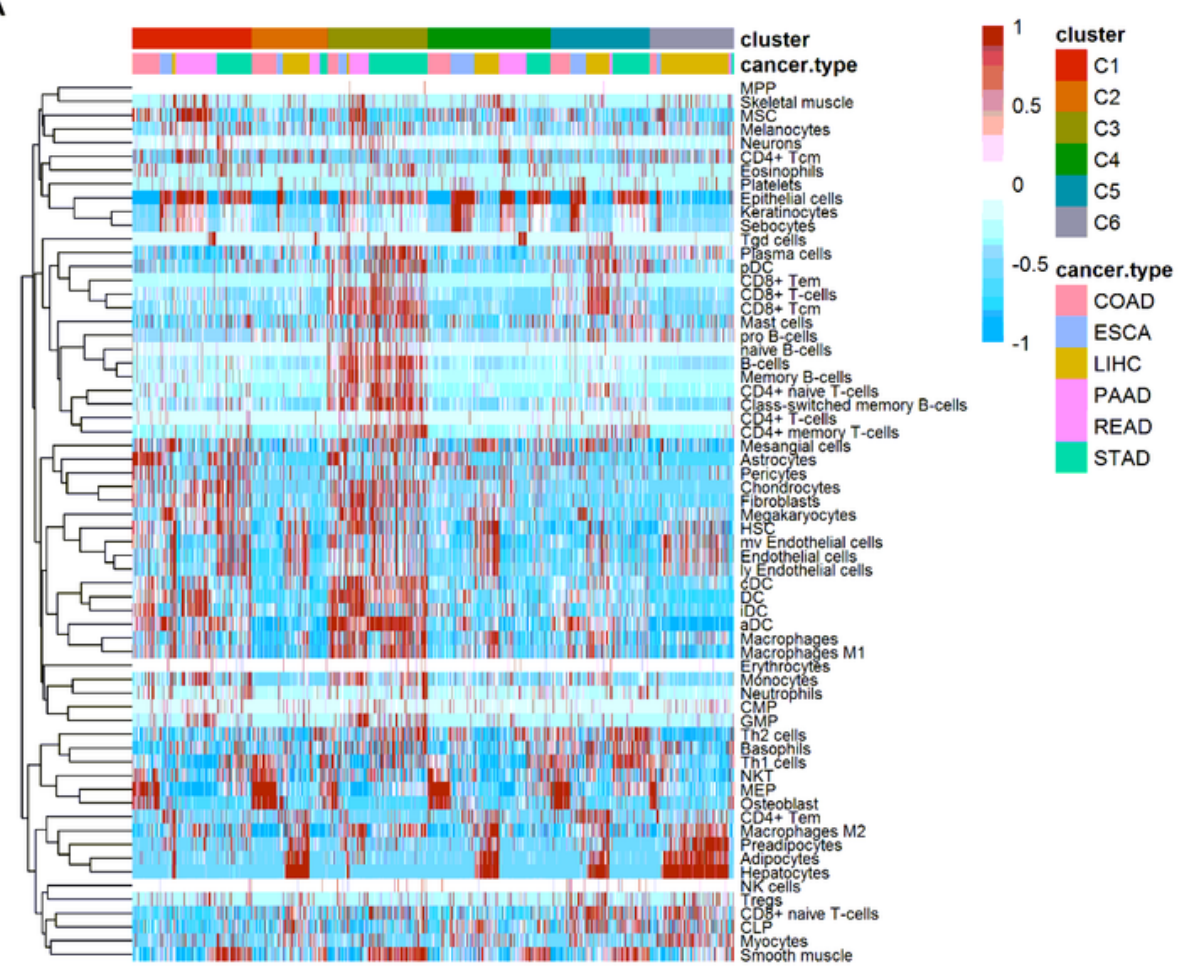

B

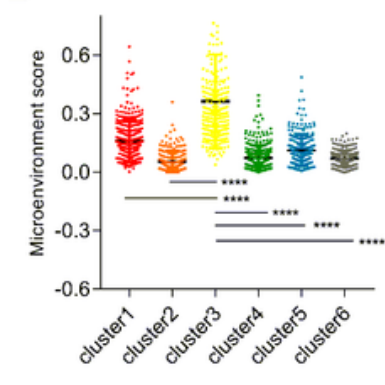

E

C

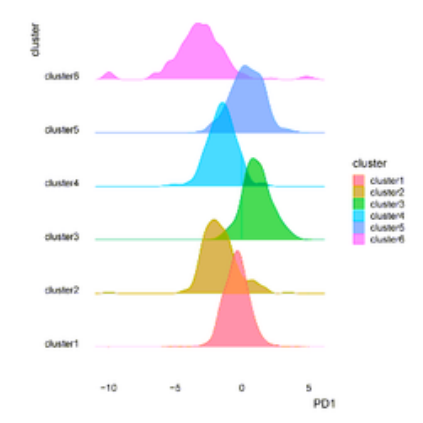

$\mathrm{F}$
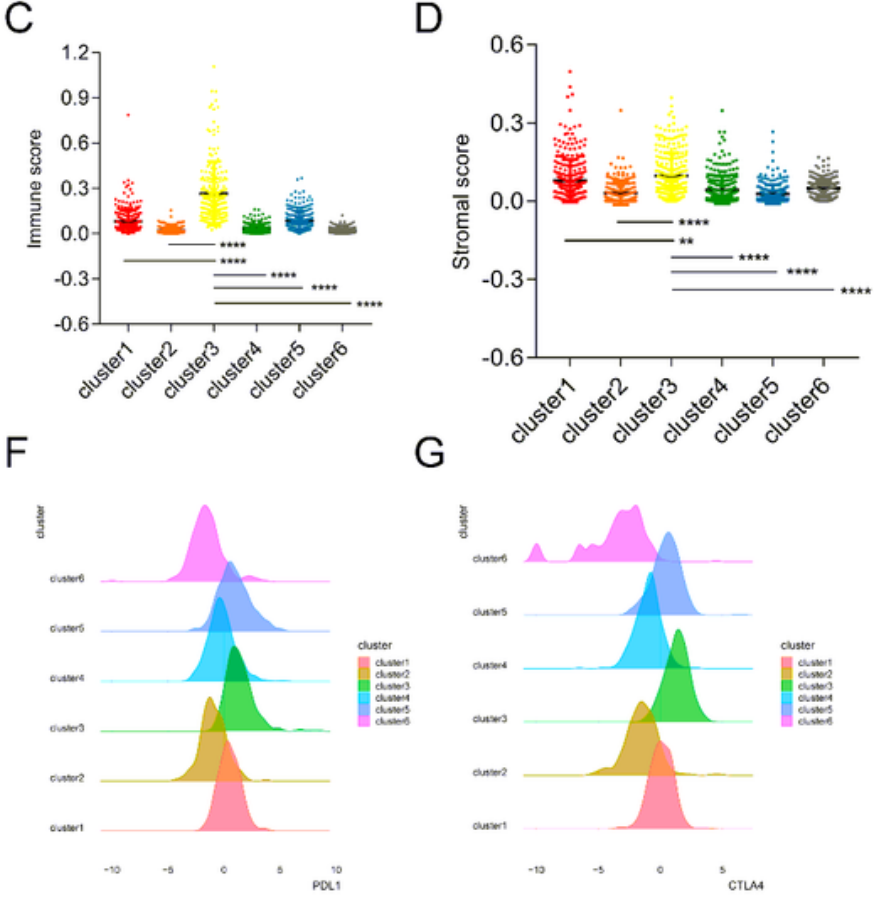

G

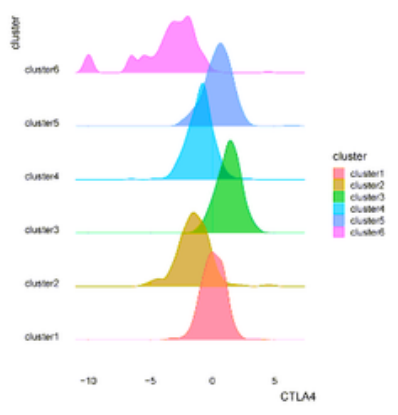

\section{Figure 5}

Cluster 3 is closely related to the characteristics of the immunosuppressive microenvironment. (A) The microenvironmental components of these six clusters conducted by $\mathrm{X}$ cell method. (B-D) The microenvironment score, immune score and stromal score in cluster 3 are significantly higher than other subgroups. (E-G) Classical immune checkpoints such as PD1, PDL1 and CTLA4 in cluster 3 and cluster 5 subgroups were significantly increased. 
A

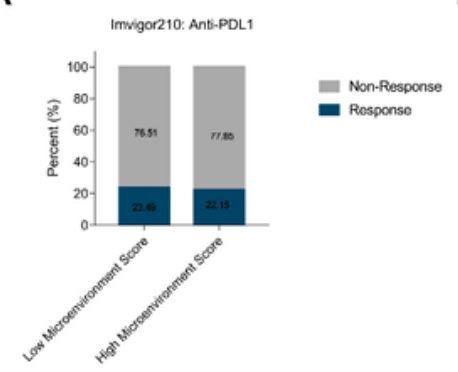

D

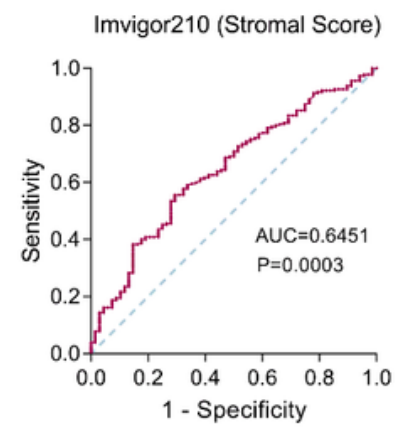

F

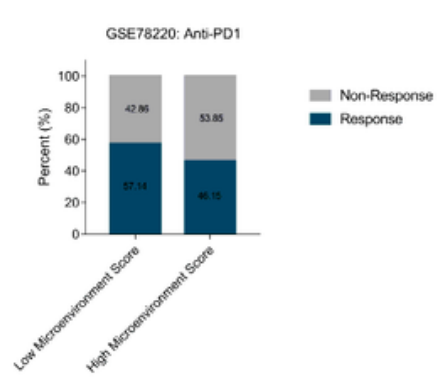

I

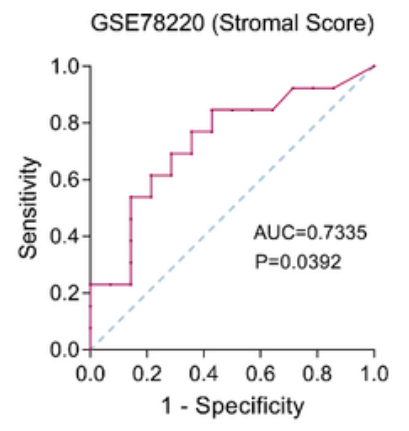

B

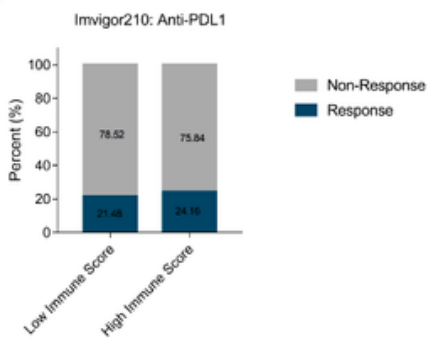

C

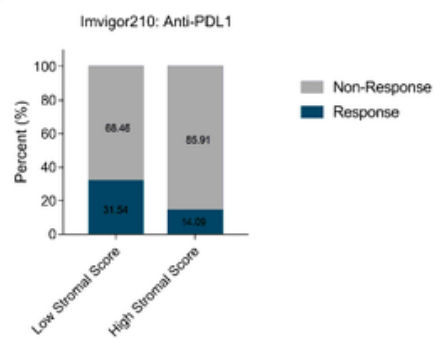

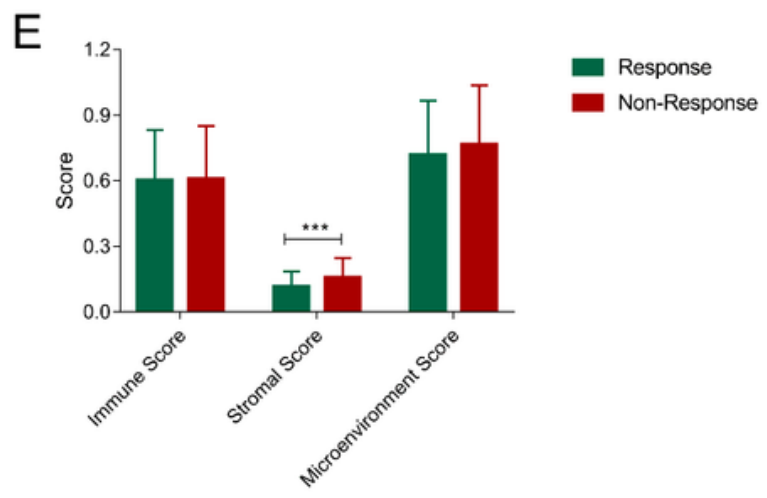

G

$\mathrm{H}$
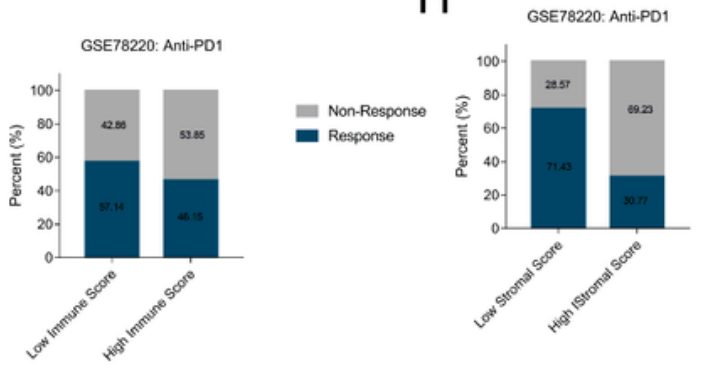

드 Non-Response

- Response

J

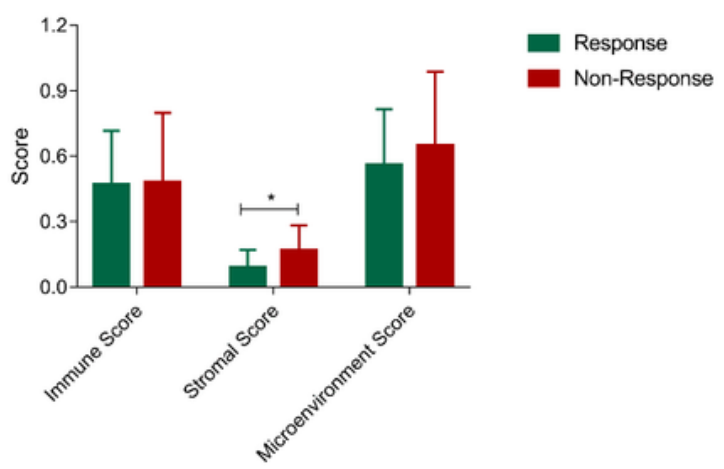

\section{Figure 6}

Stromal score can be used to predict the effect of anti-PD1/PDL1 treatment. (A-B) Patients were stratified by the microenvironment score and immune score, while there is no significant difference in the immunotherapy response rate between the high and low score groups. (C) The immunotherapy response rate in the high stromal score group was significantly lower than that in the low stromal score group. (D) The ROC curve showed that the stromal score had a well predictive effect on the positive response rate. 
(E) The stromal score in the positive response group was significantly lower than non-response group. (F$\mathrm{H}$ ) Among microenvironmental score, immune score and stromal score, only stromal score has the value in distinguishing patients with positive treatment response in GSE78220 cohort. (I) The ROC curve showed that the stromal score had a well predictive effect on the positive response rate in GSE78220 cohort. $(J)$ The stromal score in the positive response group was significantly lower than non-response group in GSE78220 cohort. 